\title{
COVID-19: a review
}

\author{
Irappa Madabhavi ${ }^{1}$, Malay Sarkar ${ }^{2}$, Nagaveni Kadakol ${ }^{3}$ \\ ${ }^{1}$ Department of Medical and Pediatric Oncology and Hematology, Kerudi Cancer Hospital, Bagalkot; ${ }^{2}$ Department of \\ Pulmonary Medicine, Indira Gandhi Medical College, Shimla; ${ }^{3}$ Department of Computer Science, Basaveshwar \\ Engineering College, Bagalkot, India
}

\begin{abstract}
The enduring epidemic outbreak which started in Wuhan city of China, in December 2019 caused by the 2019 novel coronavirus (COVID- 19) or the severe acute respiratory syndrome coronavirus 2 (SARS-CoV-2), has created a dangerous and deadly Public Health disaster of International apprehension, with cases confirmed in several countries. This novel community health trouble is frightening the universe with clinical, psychological, emotional, collapse of health system and economical slowdown in each and every part of the world infecting nearly 200 countries. A highly virulent and pathogenic COVID-19 viral infection with incubation period ranging from two to fourteen days, transmitted by breathing of infected droplets or contact with infected droplets, belongs to the genus Coronavirus with its high mutation rate in the Coronaviridae. The likely probable primary reservoir could be bats, because genomic analysis discovered that SARSCoV-2 is phylogenetically interrelated to SARS-like bat viruses. The transitional resource of origin and transfer to humans is not known, however, the rapidly developing pandemic has con-
\end{abstract}

Correspondence: Dr Irappa Madabhavi, Department of Medical and Pediatric Oncology, Kerudi Cancer Hopital, Bagalkot, Karnataka, India. E-mail: irappamadabhavi@gmail.com

Contributions: All the authors made a substantive intellectual contribution. All the authors have read and approved the final version of the manuscript and agreed to be accountable for all aspects of the work.

Conflict of interest: The authors declare that they have no competing interests, and all authors confirm accuracy.

Funding: none.

Key words: Coronavirus; COVID-19; outbreak; infection; pneumonia; ARDS; multiorgan failure; pathogenesis, cytokine storm.

Received for publication: 7 April 2020.

Accepted for publication: 7 May 2020.

${ }^{\circ}$ Copyright: the Author(s), 2020

Licensee PAGEPress, Italy

Monaldi Archives for Chest Disease 2020; 90:1298

doi: 10.4081/monaldi.2020.1298

This article is distributed under the terms of the Creative Commons Attribution Noncommercial License (by-nc 4.0) which permits any noncommercial use, distribution, and reproduction in any medium, provided the original author(s) and source are credited. firmed human to human transfer. Approximately 1,016,128 reported cases, 211,615 recovered cases and 53,069 deaths of COVID-2019 have been reported to date (April 2, 2020). The symptoms vary from asymptomatic, low grade pyrexia, dry cough, sore throat, breathlessness, tiredness, body aches, fatigue, myalgia, nausea, vomiting, diarrhea, to severe consolidation and pneumonia, acute respiratory distress syndrome (ARDS) and multiple organ dysfunction leading to death with case fatality rate ranging from 2 to $3 \%$. The most common diagnostic methods are molecular methods as RT-PCR (reverse transcription) or realtime PCR, which are prepared by means of RNA from respiratory samples such as oropharyngeal swabs, sputum, nasopharyngeal aspirate, deep tracheal aspirate, or bronchoalveolar lavage. Treatment is essentially supportive; as there is no documented clinically established and accepted antiviral remedy or vaccine existing, but some wide-ranging antiviral, anti-malarial and antiparasitic drugs have been evaluated to be used in COVID-19. Prevention includes home segregation and quarantine of suspected cases or those with mild illnesses and stringent infection control measures should be taken at hospitals to contain contact and droplet infections. International health authorities are focusing on rapid diagnosis of cases, tracing of contacts, isolation of patients and searching for antiviral therapies to counter the deadliest COVID-19 disease.

\section{Introduction}

In December 2019 China faced a deadly respiratory illness caused by a novel coronavirus, nominated as SARS-CoV-2, and has warranted an international epidemic outburst of respiratory disease called as COVID-19. Coronaviruses (CoV) comes under the genus coronavirus of the Coronaviridae family in the Nidovirales order. All CoVs are pleomorphic RNA viruses characteristically containing crown-shaped peplomer spikes on the external surface of the virus; as a consequence, it is called as a coronavirus with $80-160 \mathrm{nM}$ in size and $27-32 \mathrm{~kb}$ positive polarity [1]. Recombination rates of CoVs are very high because of constantly developing transcription errors, high mutation rates and RNA Dependent RNA Polymerase (RdRP) jumps. This new 2019$\mathrm{nCoV}$, which is a new novel strain that has not at all been previously documented in humans, has aroused a state of apprehension globally [2]. The complete spectrum of Covid-19 ranges from mild, self-limiting respiratory tract disease to ruthless progressive pneumonia, multiple organ dysfunction, and death [3-6]. As per one of the Wikipedia and the World Health Organization (WHO), $10,16,128$ cases of COVID-19 were reported globally, as of April 2, 2020, including 245,175 (6059 deaths) from USA, 115,242 (13915 deaths) from Italy, 112,065 (10,348 deaths) from Spain, 
84,789 (1109 deaths) from Germany, 81,620 cases (3322 deaths) from China, 58,441 (5380 deaths) from France, 50,468 (3160 deaths) from Iran, 33,718 (2921 deaths) from UK, and rest of the cases and deaths from 200 other countries/territories/ areas [7]. Initially, all of the cases detected in countries other than China were linked to infected cases from China, with subsequent generation of cases in Italy, USA, Japan, South Korea, Iran and France latest being involving almost 200 countries of the whole world. In the current review, we will try to summarize, history, epidemiology, origin, transmission, etio-pathogenesis, clinical symptoms, laboratory findings, diagnosis, imaging features, treatment, protection and prevention of COVID-19 infection. In view of the fact that information about this $2019-\mathrm{nCoV}$ is quickly developing; readers are informed to keep updated themselves on a regular basis.

\section{History)}

CoVs are minute, enveloped, + sense, ssRNA viruses, of dimensions varying from, 26 to $32 \mathrm{kbs}$ in length, 60 to $140 \mathrm{~nm}$ in diameter, with peptameric spiky projections on its external surface, looking as crown like spikes when we look under the electron microscope [8]. Coronavirus family subgroups mainly include alpha $(\alpha)$, beta $(\beta)$, gamma $(\gamma)$ and delta $(\delta)$ coronavirus. Previously researchers thought coronaviruses transmit a disease to only animals until the world witnessed a SARS epidemic outburst caused by SARS-CoV, 2002 in Guangdong, China and these zoonotic that are present in humans and various animals with a broad variety of clinical features from asymptomatic course to requirement of hospitalization in the intensive care unit; causing infections in respiratory, gastrointestinal, hepatic and neurologic systems [9].

During 2002-2003, when a novel SARS-CoV of the $\beta$ genera caused SARS with starting point and reservoirs as bats, transmitted to humans via the conciliator host of palm civet cats, before being controlled it infected 8422 people by and large in China and Hong Kong and caused 916 deaths with fatality rate of $11 \%$. In 2012, soon after a decade, one more highly pathogenic $\mathrm{CoV}$ infecting 2494 people, of bat origin with dromedary camels as the intermediary host, identified as Middle East respiratory syndrome coronavirus (MERSCoV), emerged in Saudi Arabia to cause an endemic in Middle Eastern countries, with 858 deaths and fatality rate of $34 \%$ [10].

Recently in December 2019, the 2019 novel Coronavirus (nCoV), which is another dangerous pandemic public health problem, has emerged in the Chinese Huanan Seafood Market of Wuhan state of Hubei Province, where livestock animals are sold and traded, and has been the center of worldwide interest owing to a pneumonia epidemic of unknown cause, which killed more than 1800 and infected over 70,000 individuals within the first 50 days of the outbreak [11,12]. Chinese researchers on January 7, 2020, reported this new virus to be a member of the $\beta-\mathrm{CoV}$ group and called as Wuhan or 2019 novel coronavirus (2019-nCov). This virus was named as $2019-\mathrm{nCoV}$ by WHO and the International Committee on Taxonomy of Viruses (ICTV) on January 12 and the disease as COVID-19 on 11 February 2020. Till to date of this writing, 2019-nCoV infected 1,016,128 individuals spreading across more than 200 countries with fatality rate of $2.9 \%$ and this signifies that the higher transmission rate of SARS-CoV-2 than SARS-CoV and the rationale might be due to genetic reassortment, and recombination incident at $\mathrm{S}$ protein in the RBD section of SARS-CoV-2.

\section{Epidemiology}

In China's Wuhan city a female shrimp seller, the coronavirus "patient zero", has made a full recovery in January after monthlong treatment. All ages are vulnerable to this lethal virus infection, it is transmitted in the course of coughing and sneezing, through the generation of large infected droplets by symptomatic patients and be able to spread about 1-2 $\mathrm{m}$ and deposit on surfaces nevertheless can also occurs from asymptomatic inhabitants before the commencement of symptoms. In one study the mode of transmission of the virus is by close contact with family members in $89 \%$ or a history of contact to the epidemic locale in $33 \%$; and $22 \%$ patients had both exposures. In both symptomatic and asymptomatic people without any difference, researchers have discovered that elevated viral loads in the nasal cavity as compared to the throat with median duration of viral shedding in Covid-19 is 20 days in patients with severe illness and could be as long as 37 days with some people as super spreaders as seen in a UK citizen who attended a convention in Singapore and contaminated 11 other people while staying in a resort in the French Alps. Patients can be contagious for as long as the symptoms last and even on clinical recuperation $[13,14]$. Similar to the SARS epidemic outburst, this outburst has occurred during the Spring Carnival in China, which is the most famous traditional festival in China, during which nearly 3 billion people travel countrywide. These conditions caused favorable circumstances for the transmission of this decidedly lethal contagious disease and severe difficulties in prevention and control of the epidemic.

In favorable atmospheric conditions this virus can stay viable on surfaces for days but are ruined in less than a minute by universal disinfectants like sodium hypochlorite, hydrogen peroxide etc. Infection is acquired either by breathing or touching surfaces contaminated by infected droplets and then touching the nose, mouth and eyes. Some researchers hypothesized that virus can also present in the stool and pollution of the water supply and subsequent transmission via aerosolization or feco oral route [14]. As per existing information, transplacental transmission from pregnant women to their fetus has not been described but, neonatal disease due to post-natal spread is documented [15]. The incubation period varies from two to fourteen d (median $5 \mathrm{~d}$ ). Due to unremitting increase of cases exponentially, modeling studies report an epidemic doubling time of 1.8 days. The basic case reproduction rate (BCR) is estimated to range from 2 to 6.47 in a variety of modeling studies as compared to; the BCR of SARS was 2 and 1.3 for pandemic flu H1N1 2009 [16]. As of 2 April 2020, over 1,016,128 cases of COVID-19 have been confirmed worldwide, having been diagnosed in 202 countries, in six continents according to an online virus tracker created by the medical journal The Lancet, and hosted by the Johns Hopkins University. There are 13 countries with $>10,000$ confirmed cases and 38 countries with between 1000 and 10,000 confirmed cases. At the time of preparing this manuscript, the World Health Organization (WHO) reported 969,289 confirmed cases including 115,000 severe cases and 50,430 death cases in around 200 countries (Table 1).

\section{Origin, emergence and spread of coronaviruses}

In 2003, Chinese population of Guangdong province infected with a SARS-CoV which caused atypical pneumonia symptoms with a diffused alveolar injury which lead to acute respiratory dis- 
tress syndrome (ARDS) and Severe Acute Respiratory Syndrome (SARS) and this was established to be a member of the Beta-coronavirus subgroup [17,18]. SARS in the beginning emerged in Guangdong, China and then spread quickly around the world infecting more than 8000 persons. A decade soon after SARS in 2012, a couple of Saudi Arabian nationals were diagnosed to be infected with another $\mathrm{CoV}$ and later confirmed as a member of Beta-CoV subgroup which is phylogenetically different from other human-CoV, named as the Middle East Respiratory Syndrome Coronavirus (MERS-CoV). MERS-CoV contaminated more than 2428 persons and 838 deaths, which initiates from a mild upper respiratory injury which on progression leads to severe respiratory disease, pneumonia, followed by ARDS and renal failure [19,20].

In December 2019, Chinese government clued-up WHO, about numerous cases of severe pneumonia epidemic with strange and unusual etiology, in adults of Wuhan, Hubei province, a capital city and major transportation hub of China. This epidemic was initiated and had universal exposure from the Hunan wholesale seafood market which frequently sold and trades live animals like bats, frogs, snakes, birds, marmots and rabbits, in Wuhan city of China [21].

The surveillance system (put into place after the SARS epidemic) was activated and respiratory samples of patients were sent to reference labs for etiologic investigations. On December $31^{\text {st }} 2019$, China notified the epidemic to the WHO and on $1^{\text {st }}$ January the Huanan sea food market was blocked. On $7^{\text {th }}$ January virus was recognized and confirmed to be $\mathrm{CoV}$ that had $>95 \%$ homology with the bat $\mathrm{CoV}$ and $>70 \%$ similarity with the SARS-CoV, from the isolates of the patients and even ecological samples from sea food market by sequence-based analysis [22]. However, auxiliary investigations discovered that some persons contracted the illness even with no documentation of visiting the seafood bazaar and number of cases started greater than ever exponentially, indicative of the fact that human-tohuman $(\mathrm{HH})$ transmission was taking place, which was consequently reported in around 200 countries around the globe. This HH scattering of the virus occurs due to close contact with contaminated individual, exposed to coughing, sneezing, respiratory droplets or aerosols and these aerosols can penetrate the human body (lungs) via breathing through the nose or mouth.

\section{Virology-pathogenesis}

Coronaviruses are viruses whose genome structure is best known among all RNA viruses. Two-thirds of RNA they have encodes viral polymerase (RdRp), RNA synthesis materials, and two large nonstructural polyproteins that are not concerned in host reaction modulation (ORF1a ORF1b). The other one-third of the genome encodes four structural proteins (spike (S), envelope (E), membrane $(\mathrm{M})$, nucleocapsid $(\mathrm{N})$, and the other helper proteins [23]. The pathogenesis of COVID-19 is inadequately understood, but analogous pathogenic mechanisms of SARS-CoV and MERS$\mathrm{CoV}$ can still give us a lot of information on the pathogenesis of SARS-CoV-2 infection. With the evolving understanding of the natural history of COVID-19 infection, it was assumed that all infections would go through an asymptomatic stage lasting three days on an average, followed by a symptomatic stage, also lasting three days on an average. Previous work has shown that the extent of transmission that occurs before symptoms develop can be an important factor in the feasibility of control [24]. The various steps of pathogenesis of coronaviruses will be described in the following paragraphs.
Table. 1. Number of cases and deaths of COVID-19 outbreak according to World Health Organization (WHO) situation report-73 on 2 April 2020.

\begin{tabular}{|c|c|c|c|}
\hline No & Country & Total cases & Deaths \\
\hline 1 & United States of America & 211,405 & 4842 \\
\hline 2 & Italy & 115,356 & 13,884 \\
\hline 3 & Spain & 109,855 & 9917 \\
\hline 4 & China & 82,817 & 3332 \\
\hline 5 & Germany & 79,678 & 1012 \\
\hline 6 & France & 61,045 & 4524 \\
\hline 7 & Iran & 50,580 & 3174 \\
\hline 8 & United Kingdom & 33,802 & 3275 \\
\hline 9 & Switzerland & 18,032 & 383 \\
\hline 10 & Turkey & 17,822 & 340 \\
\hline 11 & Belgium & 15,153 & 951 \\
\hline 12 & Netherlands & 14,633 & 1307 \\
\hline 13 & Austria & 11,240 & 166 \\
\hline 14 & Canada & 10,315 & 121 \\
\hline 15 & South Korea & 10,065 & 173 \\
\hline 16 & Portugal & 9059 & 214 \\
\hline 17 & Brazil & 6855 & 243 \\
\hline 18 & Israel & 6053 & 21 \\
\hline 19 & Sweden & 5459 & 298 \\
\hline 20 & Denmark & 5254 & 118 \\
\hline 21 & Australia & 5245 & 22 \\
\hline 22 & Norway & 4883 & 36 \\
\hline 23 & Czechia & 3867 & 47 \\
\hline 24 & Ireland & 3659 & 99 \\
\hline 25 & Chile & 3324 & 20 \\
\hline 26 & Russia & 3217 & 31 \\
\hline 27 & Malaysia & 3050 & 47 \\
\hline 28 & Poland & 2797 & 53 \\
\hline 29 & Romania & 2675 & 101 \\
\hline 30 & Japan & 2590 & 57 \\
\hline 31 & Pakistan & 2543 & 36 \\
\hline 32 & Philippines & 2538 & 104 \\
\hline 33 & Ecuador & 2504 & 217 \\
\hline 34 & Luxembourg & 2460 & 35 \\
\hline 35 & Saudi Arabia & 1877 & 22 \\
\hline 36 & Indonesia & 1825 & 188 \\
\hline 37 & Thailand & 1771 & 12 \\
\hline 38 & India & 1636 & 38 \\
\hline 39 & Peru & 1581 & 24 \\
\hline 40 & Finland & 1508 & 17 \\
\hline 41 & Dominican Republic & 1459 & 63 \\
\hline 42 & Greece & 1436 & 51 \\
\hline 43 & South Africa & 1407 & 5 \\
\hline 44 & Panama & 1373 & 36 \\
\hline 45 & Mexico & 1336 & 30 \\
\hline 46 & Iceland & 1305 & 2 \\
\hline 47 & Serbia & 1220 & 13 \\
\hline 48 & Argentina & 1142 & 30 \\
\hline 49 & Algeria & 1110 & 81 \\
\hline 50 & Singapore & 1074 & 3 \\
\hline 51 & Croatia & 1059 & 6 \\
\hline 52 & Columbia & 1014 & 18 \\
\hline
\end{tabular}




\section{Coronavirus entry and replication}

SARS-CoV-2 possesses and expresses the characteristic coronavirus makeup proteins consisting of peptameric spike protein, polyproteins, nucleoproteins, and membrane proteins, such as RNA polymerase, 3-chymotrypsin-like protease, papain-like protease, helicase, glycoprotein, and accessory proteins [25]. S protein of $\mathrm{CoV}$ has been considered as a noteworthy determinant of virus ingress into host cells. The glycoprotein spikes on the external surface of coronaviruses are accountable for the affection, obligatory binding and ingress of the virus to host cells, through its cellular receptors, like ACE2 for SARS-CoV and SARS-CoV-2, CD209L (a C-type lectin, also called L-SIGN) for SARS-CoV, and DPP4 for MERS-CoV [26]. The spike protein of SARS-CoV-2 contains a 3-D structure in the RBD region, the 394 glutamine residues in that region of SARS-CoV-2 are acknowledged by the critical lysine 31 residues on the human ACE2 receptor [27].

The entry of SARS-CoV into host cells was primarily recognized to be able by direct membrane fusion between the virus and plasma membrane [28]. This direct membrane fusion of SARS$\mathrm{CoV}$ is due to critical proteolytic cleavage event in $\mathrm{S}$ protein at position (S20), which mediates the membrane fusion and viral infectivity, the clathrin-dependent and -independent endocytosis mediated entry pathways [29]. After entering the cells, the RNA genome of virus is released into the cytoplasm and is translated into two polyproteins and structural proteins, subsequent to which the viral genome starts to replicate. The freshly created envelope glycoproteins are inserted into the membrane of the endoplasmic reticulum or Golgi, and the nucleocapsid is formed by the combination of genomic RNA and nucleocapsid protein. Viral particles germinate into the endoplasmic reticulum-Golgi intermediate compartment (ERGIC) and the vesicles containing the virus particles then combine with the plasma membrane to discharge the virus.

\section{Antigen presentation in coronavirus infection}

Antigen presentation cells (APC), which are fundamental part of the body's anti-viral immunity recognizes the antigens and antigenic peptides released by the virus while entering the cells. Virusspecific cytotoxic $\mathrm{T}$ lymphocytes (CTLs) recognize the antigenic peptides which are presented by major histocompatibility complex (MHC; or human leukocyte antigen (HLA) in humans) mainly MHC-I. CD4+ T cells promote the production of virus-specific antibodies by activating T-dependent $\mathrm{B}$ cells. CD8 $+\mathrm{T}$ cells are cytotoxic and can kill viral infected cells and account for about $80 \%$ of total infiltrative inflammatory cells in the pulmonary interstitium in SARS-CoV-infected patients and play a vital role in clearing CoVs in infected cells and inducing immune injury. Regrettably, there is still lack of any statement about it, and we can only get some information from previous researches on SARS$\mathrm{CoV}$ and MERS-CoV [30]. In MERS-CoV infection, MHC II molecules, such as HLA-DRB1*11:01 and HLA-DQB1*02:0, are related with the susceptibility to MERS-CoV infection [31].

\section{Humoral and cellular immunity}

Presentation of antigens and antigenic peptides to immune cells consequently stimulates and switches on the body's humoral and cellular immune system, which are mediated by virus-specific $\mathrm{B}$ and $\mathrm{T}$ cells. The complement system plays a vital role in the host immune response by detecting and responding to foreign antigens of $\mathrm{CoV}$ infection. Antibody profile against SARS-CoV virus has a characteristic representative pattern of $\operatorname{IgM}$ and $\operatorname{IgG}$ production; SARS-specific IgM antibodies disappear at the end of week 12, while the IgG antibody can last for a long time, which indicates
IgG antibody mainly acts as a protective role, which are principally S-specific and Nspecific antibodies [32]. T cells, CD4+ T cells, and CD8 $+\mathrm{T}$ cells predominantly play a considerable momentous antiviral role by balancing the combat against pathogens and the risk of developing autoimmunity or overwhelming inflammation. The most recent research on cellular immunity shows the number of $\mathrm{CD}^{+}$and $\mathrm{CD}^{+} \mathrm{T}$ cells in the peripheral blood of SARS-CoV2-infected patients is considerably reduced. However its status of excessive activation, is evidenced by sky-scraping proportions of HLA-DR (CD4 3.47\%) and CD38 (CD8 39.4\%) double positive fractions. Even if there is no antigen, $\mathrm{CD} 4^{+}$and $\mathrm{CD} 8^{+}$memory $\mathrm{T}$ cells can endure for four years in a part of SARS-CoV recovered individuals and can carry out $\mathrm{T}$ cell proliferation, DTH reaction and production of IFN-g and this conclusion may endow with worthless information for the cogent design of vaccines against SARS-CoV-2 [33].

\section{Cytokine storm or sHLH in COVID-19}

Secondary haemophagocytic lymphohistiocytosis (sHLH) most commonly triggered by viral infections is an under-recognized, hyper inflammatory syndrome characterized by a fulminant and fatal hypercytokinaemia with multiorgan failure in COVID-19 infection. Most of the recent studies show ARDS is the common immunopathological event and most important cause of death in SARS-CoV-2, SARS-CoV and MERS-CoV infections [3]. Principal features of sHLH comprise of unrelenting fever, cytopenias, and hyperferritinaemia; pulmonary involvement (including ARDS) occurs in approximately $50 \%$ of patients. In one recent report of the 41 SARS-CoV-2-infected patients admitted in the early stages of the outburst, six succumbed to death from ARDS. ARDS in SARS-CoV infection occurs due to cytokine storm, the fatal unrestrained systemic inflammatory response resulting from the release of large amounts of pro-inflammatory cytokines with cytokine profile resembling sHLH, characterized by increased interleukin (IL)-2, IL-1b, IL-6, IL-7, IL-12, IL-18, IL-33, granulocyte-colony stimulating factor, interferon- $\gamma$ and $\alpha$ inducible protein 10 , monocyte chemoattractant protein 1 , macrophage inflammatory protein $1-\alpha$, and tumor necrosis factor- $\alpha$ and TGF $\beta$, etc. and chemokines (CCL2, CCL3, CCL5, CXCL8, CXCL9, CXCL10, etc.) by the immune effector cells [34]. Similar to those with SARS-CoV-2, SARS-CoV, individuals with severe MERS-CoV infection shows cytokine storm with elevated levels of IL-6, IFNa, and CCL5, CXCL8, CXCL-10 which triggers a brutal assault by the immune system to the body, leading to ARDS, multi organ dysfunction, and finally death [35].

\section{Coronavirus immune evasion}

SARS-CoV and MERS-CoV uses numerous strategies to avoid immune responses against the virus and proteins by the host cells whose immune response inhibits virus replication, promotes virus clearance, induces tissue repair, and triggers a prolonged adaptive immune response against the viruses. SARS-CoV and MERS-CoV can induce the production of double-membrane vesicles that lack the evolutionarily conserved microbial structures called pathogenassociated molecular patterns (PAMPs) which are recognized by pattern recognition receptors (PRRs) and then reproduce in these vesicles, thereby evading the host detection of their dsRNA [36]. IFN-I (IFN- $\alpha$ and IFN- $\beta$ ) has a defensive effect on SARS-CoV and MERS-CoV infection, but this pathway is inhibited in infected mice by the host cells. Accessory protein 4a of MERSCoV may block the induction of IFN, and ORF4a, ORF4b, ORF5, and membrane proteins of MERSCoV may stall nuclear transport of IFN 
regulatory factor 3 (IRF3) and activation of IFN b promoter. The antigen presentation in MERS-CoV infection can also be affected by the coronavirus especially by the down-regulation of gene expression related to antigen presentation [37]. Hence, destroying the immune evasion of SARSCoV-2 is very important and crucial in its treatment and specific drug discovery.

\section{Clinical symptoms}

The symptoms of COVID-19 infection appear following an incubation period of roughly 5.2 days. The period from the onset of COVID-19 symptoms to death ranged from 6 to 41 days with a median of 14 days. The median time period in days (d) from commencement of symptoms, to dyspnea is $5 \mathrm{~d}$, to hospitalization is 7 $\mathrm{d}$ and to acute respiratory distress syndrome (ARDS) is $8 \mathrm{~d}$. This period is dependent on the age of the patient and status of the patient's immune system. It was shorter among patients $>70$-years old compared with those under the age of 70 [38]. Common symptoms at onset of COVID-19 illness are fever (85-90\%), body aches and myalgia (10-15\%), dry cough $(65-70 \%)$, running nose and nasal congestion $(<10 \%)$, sore throat $(10-15 \%)$, tiredness, fatigue (35-40\%); some people have experienced the loss of smell or taste, while other symptoms include sputum production (30-35\%), headache (10-15\%), chills (10-12\%), haemoptysis, nausea, vomiting, diarrhea $(<5 \%)$, dyspnoea $(15-20 \%)$, pleuritic chest pain, palpitations, and chest tightness. The severity of COVID-19 symptoms can range from very mild to severe disease with clinical profile constituting; no symptoms in $30 \%$, mild/moderate symptoms in $55 \%$, severe symptoms in $10 \%$ and critical symptoms in $5 \%$ cases. People who are older or who have existing chronic medical conditions, such as heart disease, lung disease or diabetes, or who have compromised immune systems may be at higher risk of serious illness [3]. Clinical features revealed by a chest CT scan presented as pneumonia, however, there were abnormal features such as RNAaemia, ARDS, acute severe cardiac damage, and incidence of grand-glass opacities that led to death [3]. In some cases, the numerous peripheral ground-glass opacities were observed in subpleural regions of both lungs that likely induced both systemic and localized immune response that led to increased inflammation. Regrettably, treatment of some cases with interferon inhalation showed no clinical effect and instead appeared to worsen the condition by progressing pulmonary opacities [39].

It is important to note that there are similarities in the symptoms between COVID-19 and earlier beta CoVs such as fever, body aches, tiredness, dry cough, dyspnea, and bilateral groundglass opacities on chest CT scans. In addition, based on results from chest radiographs upon admission, some of the cases demonstrate opacities in the upper lobe of the lung that is associated with increasing dyspnea with hypoxemia [40]. Importantly, whereas patients infected with COVID-19 developed gastrointestinal symptoms like diarrhea, a low percentage of MERS-CoV or SARS-CoV patients experienced similar GI distress. Therefore, it is important to test faecal and urine samples to rule out a possible unusual route of transmission, specifically through health care workers, patients, etc. [41]. Therefore, development of methods to identify the various modes of transmission such as feacal and urine samples are urgently warranted in order to develop strategies to inhibit and/or minimize transmission and to develop therapeutics to control the disease.

The authors of the Chinese CDC report divided the clinical manifestations of the disease by their severity:
- Mild disease: non-pneumonia and mild pneumonia; this occurred in $81 \%$ of cases.

- $\quad$ Severe disease: dyspnea, respiratory frequency $\geq 30 / \mathrm{min}$, blood oxygen saturation $\left(\mathrm{SpO}_{2}\right) \leq 93 \%, \mathrm{PaO}_{2} / \mathrm{FiO}_{2}$ ratio or $\mathrm{P} / \mathrm{F}$ [the ratio between the blood pressure of the oxygen (partial pressure of oxygen, $\mathrm{PaO}_{2}$ ) and the percentage of oxygen supplied (fraction of inspired oxygen, $\mathrm{FiO}_{2}$ ) $]<300$, and/or lung infiltrates $>50 \%$ within 24 to 48 hours; this occurred in $14 \%$ of cases.

- Critical disease: respiratory failure, septic shock, and/or multiple organ dysfunction (MOD) or failure (MOF); this occurred in $5 \%$ of cases.

\section{Complications}

Various complications in hospitalized COVID-19 patients are; ARDS in 22-5\% (range 17-29\%), ICU admission rate- 5\%, invasive or non-invasive ventilation in $6 \%$, acute cardiac injury (elevated trophonin levels, myocardial ischemia and cardiac arrest), secondary infections and sepsis, acute kidney injury (AKI), multiorgan failure in a small subgroup of severe ICU cases: secondary hemophagocytic lymphohistiocytosis (sHLH) (a cytokine storm syndrome).

\section{Diagnosis}

\section{WHO definitions}

A) Suspect case. A patient with acute respiratory illness (fever and at least one sign/symptom of respiratory disease (e.g., cough, shortness of breath), AND with no other etiology that fully explains the clinical presentation AND a history of travel to or residence in a country/area or territory reporting local transmission of COVID-19 disease during the 14 days prior to symptom onset. OR A patient with any acute respiratory illness AND having been in contact with a confirmed or probable COVID19 case (see definition of contact) in the last 14 days prior to onset of symptoms; OR A patient with severe acute respiratory infection (fever and at least one sign/symptom of respiratory disease (e.g., cough, shortness breath) AND requiring hospitalization AND with no other etiology that fully explains the clinical presentation.

B) Probable case. A suspect case for whom testing for COVID-19 is inconclusive.

C) Confirmed case. A person with laboratory confirmation of COVID-19 infection, irrespective of clinical signs and symptoms.

Clinical conclusion of COVID-19 is essentially based on epidemiological record, clinical symptoms and some supplementary examinations, such as nucleic acid detection tests, CT scan, immune identification technology (Point-of-care Testing (POCT) of $\operatorname{IgM} / \mathrm{IgG}$, enzyme-linked immunosorbent assay - ELISA) and blood culture for virus growth. On the other hand, the clinical features and signs of patients infected with SARS-CoV-2 are extremely atypical, including respiratory symptoms, dry cough, fever, dyspnea, tiredness, fatigue and viral pneumonia. For that reason, additional auxiliary examinations are essential for the identification of COVID-19.

\section{Laboratory investigations}

Laboratory investigations are frequently non specific. Typical abnormal laboratory findings were elevated creatine kinase (CPK) - MB (31\%), ESR, CRP, decreased lymphocytes (31\%), leucopenia 
(19\%), and elevated procalcitonin (17\%). Variables that are associated significantly with severity of COVID-19 were lymphopenia (decreased lymphocytes especially lymphocyte count $<1000$ ), elevated body temperature, increased creatinine, high levels of procalcitonin, D-dimer, LDH, prothrombin time, ALT/AST and CPKMB. The white cell and platelet count are usually normal or mildly low. A high procalcitonin level may indicate a bacterial co-infection. In one recent study raised $\mathrm{LDH}$ and D-dimer are associated with development of ARDS.

\section{Nucleic acid, antigen-antibody and virus detection}

The diagnostic tools are molecular methods, serology and viral culture. The two most frequently used nucleic acid detection technologies for SARS-CoV-2 are real-time quantitative polymerase chain reaction (RT-qPCR) and high-throughput sequencing. The WHO recommends RT-qPCR the most common, effective and straightforward diagnostic method which is made using RNA from respiratory specimens from both the upper respiratory tract (nasoand oropharyngeal samples) and lower respiratory tract such as expectorated sputum, endotracheal aspirate, or bronchoalveolar lavage (should be used in mechanically ventilated patients). In the laboratory, amplification of the genetic material extracted from the sample is through a reverse polymerase chain reaction (RT-PCR), which involves the synthesis of a double-stranded DNA molecule from an RNA mold for detecting pathogenic viruses in respiratory secretions. Recent papers have revealed that lower respiratory tract samples can offer significantly higher viral load and genome fraction than upper respiratory tract samples but unfortunately, we are incapable of doing sampling of lower respiratory tract secretions on a regular basis. The most respected and trustworthy detection technique for SARS-CoV-2 is virus blood culture and highthroughput sequencing of the whole genome but this technology in clinical diagnosis is limited because of its equipment dependency and high cost $[25,42,43]$. The Chinese CDC recommends the RT-q PCR that uses the specific precise primers and probes targeting different gene regions [RdRp/Helicase (Hel), Spike (S), and N] of SARS-CoV-2 were designed and tested and defined as a laboratory-confirmed infection when atleast two of the three targets are positive. Real -time RT -PCR assays for SARS-CoV-2 RNA detection were performed using QuantiNova Probe RT -PCR Kit (Qiagen) in a LightCycler 480 Real-Time PCR System (Roche, Basel, Switzerland). The recognition of SARS-CoV using real time RT-qPCR is believed to be highly specific, but with sensitivity reported as low as $60-70 \%$ and as high as $95-97 \%$, depending upon the protocol used, sample type and number of clinical specimens collected [44]. The sensitivity of antibody detection by immunological detection kits is generally lower than molecular methods and is mostly used in retrospective diagnosis [45]. The sensitivity of SARS-CoV N-based IgG ELISA (94.7\%) is considerably higher than that of SARS-CoV S-based IgG ELISA (58.9\%). Viral culture is a more time-consuming method compared to the other methods. Culture is much more useful in the first stage of outbreaks before other diagnostic methods become clinically available. In addition, viral cultures can be used and assayed in the in vitro and in vivo clinical antiviral treatment studies and vaccine evaluation trials [9].

\section{CT scans and other diagnostic methods}

Chest radiography is typically the first-line imaging modality used for patients with suspected COVID-19, may be normal in early or mild disease, abnormal in $69 \%$ of cases at the time of admission, and $80 \%$ shows abnormalities during hospitalization. The most common abnormalities are bilateral, peripheral and lower zone predominant, air space opacities, consolidation or ground glass opacities. Diagnosis of COVID-19, by RT-qPCR is specific, but its false-negative rate cannot be disregarded because of the relentless cost of missed diagnosis, hence many clinicians proposed CT scan to be used as an essential auxiliary diagnostic method. Combination of repeated RT-qPCR tests and chest CT scan may be helpful especially in patients with a high clinical suspicion of SARS-CoV-2 infection with negative RT-qPCR screening. High-resolution CT (HRCT) of chest without contrast is essential for early diagnosis and evaluation of disease severity of patients with SARS-CoV-2. The typical CT images show bilateral, peripheral and subpleural ground-glass opacities (GGO), air space consolidation, bronchovascular thickening, traction bronchiectasis and crazy paving appearance and some atypical findings includes; mediastinal lymphadenopathy, tree in bud appearance, pneumothorax, pleural effusion and multiple tiny pulmonary nodules and sometimes abnormal in asymptomatic patients without any clinical evidence of lower respiratory tract involvement. Even though CT is more sensitive and specific, on 16 March 2020, an AmericanSingaporean panel published that CT findings were not part of the diagnostic criteria for COVID-19. But abnormal CT scans can be used to diagnose COVID-19 in suspect cases with initial negativity and positivity on repeat molecular testing [46].

\section{Treatment}

No specific treatment, drugs, monoclonal antibodies (mAbs), or vaccines exist for SARS-CoV-2 till the end of March 2020 [47]. Concerted efforts are required to effect rapid diagnosis, quarantine infected cases to ensure adequate isolation to prevent transmission to other contacts, patients and healthcare workers and provide effective essential supportive and symptomatic treatment. Patients with mild illness, while maintaining good hydration and nutrition and controlling fever and cough, should be asked to manage at home with proper counseling about danger signs. Patients with hypoxia, can be managed with provision of oxygen through nasal prongs, face mask, high flow nasal cannula (HFNC) or non-invasive ventilation. In severe pulmonary dysfunction cases mechanical ventilation or an extra corporeal membrane oxygen (ECMO) support may be required. Patients with renal dysfunction may require renal replacement therapy. If any patient is having suspicious or proven co-infections; antibiotics and antifungals may be required (Figure 1). Chinese guidelines do propose short term steroid therapy with low-to-moderate dose corticosteroids in COVID-19 ARDS but it is unproven and against their use according to current international consensus and WHO $[48,49]$. Comprehensive guidelines have been published by the WHO for the management of critically ill COVID-19 patients [50].

At this time as there is no existence of specific antiviral therapies for SARS-2-CoV, the combination of the protease inhibitors, ritonavir, and lopinavir, or a triple combination of these antiviral agents with the addition of ribavirin, showed some success in the treatment of SARS, and early reports suggested similar efficacy in the treatment of COVID-19 [51]. However, a more recent randomized, controlled open-label trial failed to demonstrate any added benefit of lopinavir-ritonavir combination therapy [52]. Initially, interferons- $\alpha$ nebulization, broad-spectrum antibiotics, and antiviral drugs were used to reduce the viral load [53,54], however, only remdesivir has shown promising impact against the virus [55]. Remdesivir alone or in combination with chloroquine or Interferon beta, a drug originally developed to treat Ebola virus 


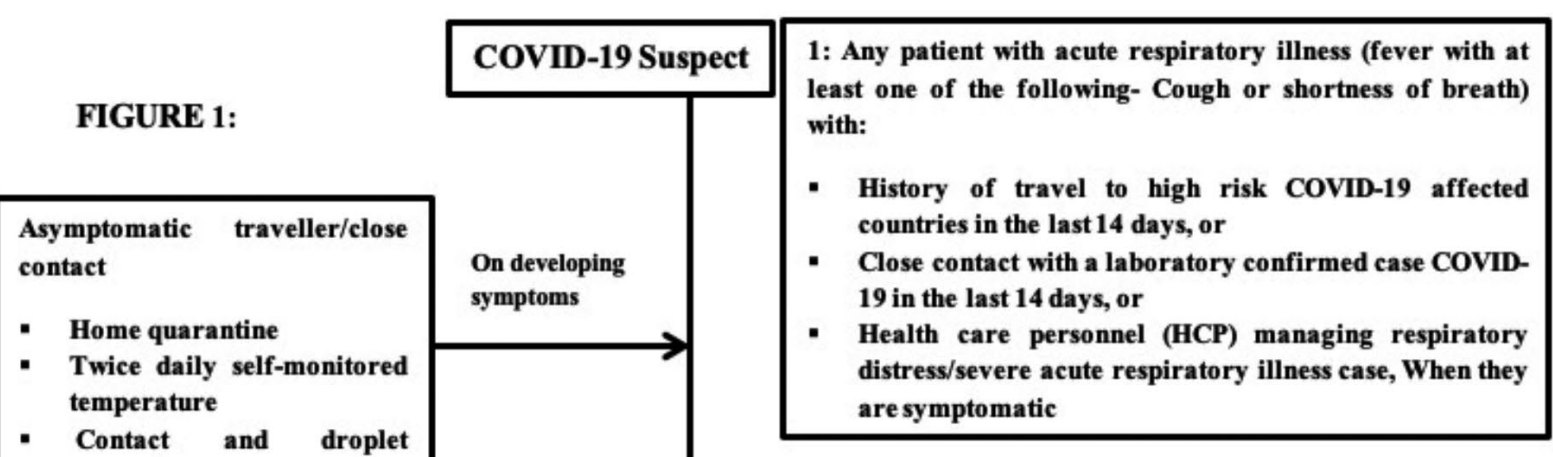

Contact and droplet

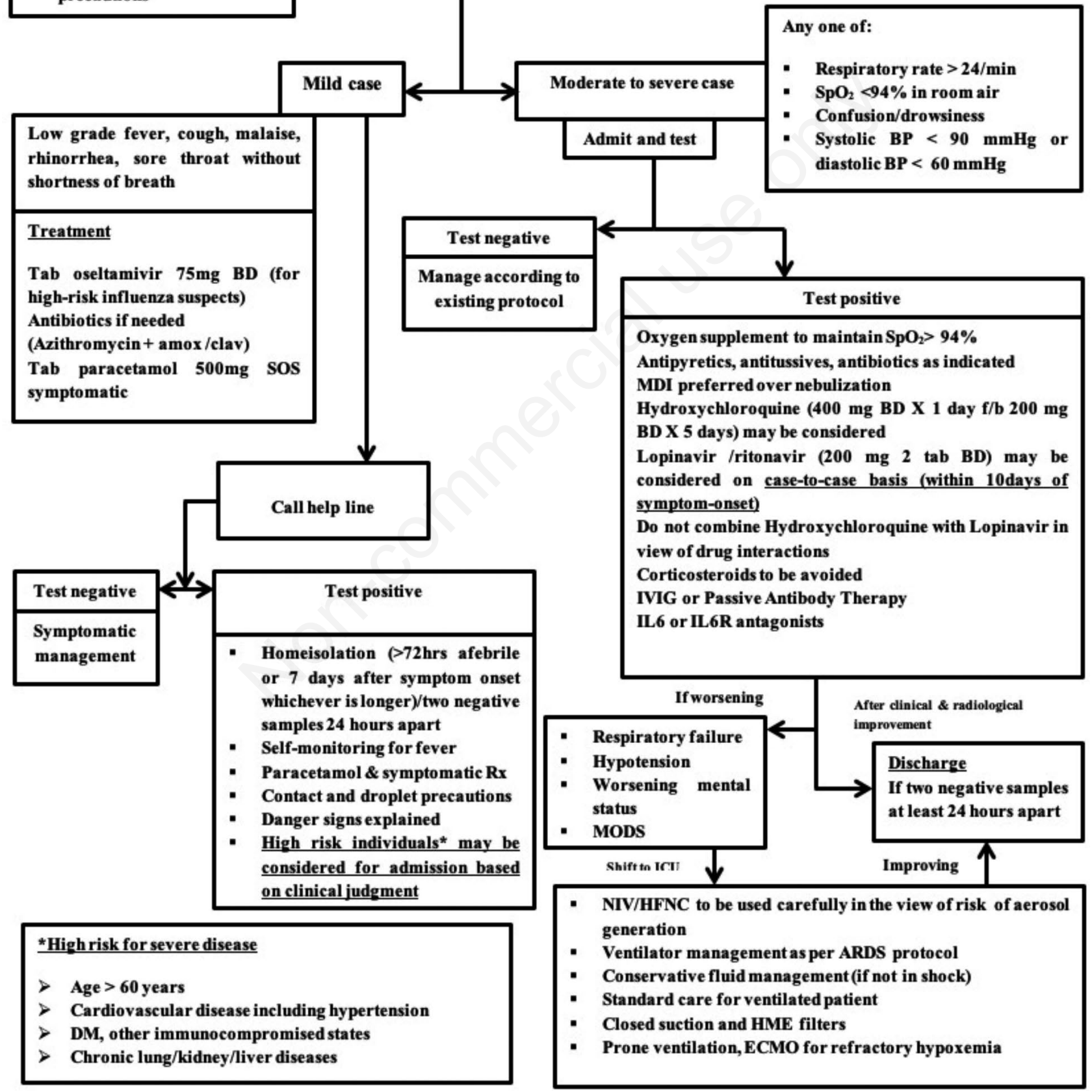

Figure 1. COVID-19 treatment flow-chart. 
and shown to be effective against MERS-CoV and SARS-CoV, showed promising in vitro results against SARS-CoV-2 and is undergoing phase III trials [51,54,56,57]. Other anti-virals which are currently being evaluated against SARS-CoV-2 infection are Oseltamivir, Nafamostat, Nitazoxanide, Ribavirin, Ivermectin, Penciclovir, Favipiravir, Lopinavir, Darunavir, Ritonavir, AAK1, Baricitinib, Cobicistat and Arbidol [54,56-58].

It is becoming apparent that the "ground glass" infiltrative appearance seen on CT scans from COVID-19 patients with pneumonitis is reminiscent of imaging from patients with immune checkpoint inhibitor (ICI)-induced pneumonitis [59]. Additionally, elevated IL-6 is a hallmark inflammatory signature seen in serum of patients with severe COVID-19 acute respiratory distress. One possibility is to encourage the use of IL-6 or IL-6-receptor blocking antibodies like tocilizumab, sarilumab, and siltuximab that are FDA-approved for various conditions including rheumatologic disease and the lymphoproliferative disorder Castleman's syndrome, cytokine release syndrome (CRS) in patients receiving CAR T cell therapy [60]. Now, data from the frontlines of the pandemic indicates that the agent may offer lifesaving benefit for COVID-19 patients with respiratory distress. Various combinations antivirals or antibiotics with traditional Chinese medicines were also evaluated against SARS-CoV-2 induced infection in humans and mice. Several therapies, such as remdesivir and favipiravir, are under investigation, but the antiviral efficacy of these drugs is not yet known [47,61].

\section{Passive antibody therapy}

Passive antibody therapy (PAT) involves the administration of neutralizing antibodies against a given agent to a vulnerable individual for the point of preventing or treating an infectious disease due to that agent. Monoclonal antibodies represent the major class of biotherapeutics for passive immunotherapy to fight against viral infection or antiviral protection by harnessing the natural power of the immune system. Whereas in active vaccination, the initiation of an immune response takes more time to develop and varies depending on the vaccine recipient. Therefore, PAT is the only solitary way of providing instantaneous urgent immunity to vulnerable persons. The use of convalescent plasma/serum or PAT which contain neutralizing antibodies to the relevant virus is not new; it was used for severe acute respiratory syndrome (SARS), pandemic 2009 influenza A (H1N1), avian influenza A (H5N1), several hemorrhagic fevers such as Ebola, and other viral infections [62]. Anticipatory mechanisms of action by which PAT in SARS-CoV-2 mediate protection, are viral neutralization by which reducing virus replication in epithelial cells and preventing virus shedding and spread to susceptible hosts, antibody-dependent cellular cytotoxicity and/or phagocytosis. Possible sources of antibody for SARS-CoV-2 are human convalescent sera from COVID-19 recovered individuals, mAbs, animal preparations, and by genetically engineered cows that create human antibody [63]. The lone antibody type that is currently accessible for immediate instantaneous use is human convalescent sera and with continuing pandemic more individuals contract COVID-19 and recover, the number of potential donors will go on to amplify.

A study from China by Shen et al. shows that administration of convalescent plasma containing neutralizing antibody to critically ill patients with COVID-19 was followed by improvement in their clinical status [64]. Recent studies show isolation of the blood plasma from clinically improved healthier patients of COVID-19 and injecting the mAbs like CR3022 which binds with the spike RBD of SARS-CoV-2 has the potential to be developed as a therapeutic candidate, alone or in combination with other neutralizing antibodies for the prevention and treatment of COVID-19 infection, in to the infected patients revealed encouraging affirmative results with rapid recovery $[65,66]$. Deploying PATs against the rapidly increasing number of COVID-19 cases provides an unprecedented opportunity to perform clinical studies of the efficacy of this treatment against SARS-CoV2.

\section{Vaccines for SARS-CoV-2}

The ongoing emergence of $\mathrm{CoVs}$ at regular intervals poses a noteworthy threat to human health and economy. Sarcastically, even after a decade of investigations on $\mathrm{CoV}$, still there are no licensed vaccines or therapeutic agents to treat coronavirus infection which highlights an urgent need to develop effective vaccines or post-exposure prophylaxis to prevent future epidemics. But previous vaccines or strategies could be used to develop an effective vaccine against SARS-CoV. Urbani (AY278741) strain, BJ01 (AY278488), Utah (AY714217), ZJ01 (AY297028), FRA (AY310120), GD01 (AY278489), HKU-39849 (AY278491), Tor2 (AY274119), NS1 (AY508724), and GZ50 (AY304495) strains, a recombinant protein unit, DNA fragment, inactivated whole virus or live-vectored strain of SARS-CoV (AY278741), when administered to mice and hamsters resulted in the production of protective neutralizing antibodies, and considerably reduced the SARS-CoV2 infection $[67,68]$. The various other agents used for vaccine preparation are, mRNA based by the US National Institute of Allergy and Infectious Diseases, INO-4800-DNA based, recombinant 2019-nCoV S protein subunit-trimer based vaccine by Clover Biopharmaceuticals and inactivated virus vaccine by the Chinese CDC [69-71].

\section{Prevention, protection and future directions}

Age old statement of prevention is better than cure holds good at this stage in view of the fact that till date there are no approved treatments, mAbs, drugs or vaccines for this infection. But a number of properties like vague non-specific clinical features of the illness, infectivity and transmission in the asymptomatic stage of the disease, protracted incubation period, tropism for mucosal surfaces such as the conjunctiva, prolonged duration of the illness and transmission even after clinical recovery of this virus make prevention difficult namely. WHO recommends home isolation or quarantine of confirmed or suspected cases with mild illness with counseling of danger signs of the disease. All the patients while on treatment should be asked to wear a simple surgical mask and advised to practice cough hygiene. Caregivers of the patient while being in the room should be asked to wear a surgical mask and use hand hygiene every 15-20 min.

Extensive measures and actions should be taken to contain the infection, like timely publication of epidemic information for elimination of infectious source, early diagnosis, reporting, isolation, supportive treatments and for avoiding unnecessary panic. CDC reminds basic measures such as hand washing, using disinfectant solutions, avoiding contact with patients in order to prevent the spread of viruses by droplets. Precautionary actions including the provision of medicines supply chains, personal protective equipment (PPE), and hospital supplies should be made in a short time for the protection of the people. Based on the SARS-CoV-2 epidemic experience, the government should take many effective measures including closing public transport, reducing migration and promoting personal protection with masks. As such there are no proven medicines which can be used as prophylactically, some people especially doc- 
tors are taking hydroxychloroquine and azithromycin as a prophylactic measure, but some developed toxicity of the drugs and precipitation of the existing co morbid conditions and 2 doctors in India died of cardiac arrest after taking these drugs.

At the individual level maximum risk of COVID-19 transmission is to healthcare workers, as they are frequently engaged in treating the infected patients, even the doctor who first warned about the virus to the whole world has died, too [72]. It is essential to save from harm to the healthcare workers to ensure continuity of health care for the needy people and to prevent transmission of infection to other patients and hospital staff and healthcare workers should be provided with fit tested N95 respirators, protective suits and goggles. All the infected patients should be placed in separate rooms or cohorted together, rooms, surfaces and equipment must undergo standard decontamination and sanitization preferably with sodium hypochlorite. Airborne transmission by aerosol generating procedures such as intubation, suction and tracheostomies can occur and precautions should be taken like wearing protective goggles, masks and PPE. Patients can be discharged from the isolation ward once they are afebrile for atleast $3 \mathrm{~d}$ and have two consecutive negative molecular tests at $1 \mathrm{~d}$ sampling interval. All contacts including healthcare workers should be monitored for development of symptoms of COVID-19 [73].

At the community level, all the people should be educated about the symptoms of COVID-19, through pamphlets, social media, television or websites, should be asked to avoid overcrowded areas like market, bazaar, social functions, gatherings and entertainment parks and should be asked to postpone non-essential travel to places with ongoing transmission. People should be educated and asked to practice proper cough hygiene by coughing in sleeve/tissue rather than hands to prevent spread of droplets and practice hand hygiene by hand washing with soaps and sanitizers frequently every 15-20 min. All the patients with respiratory symptoms should be asked to use surgical masks to avoid droplet spreading to the others and contamination of the surfaces. The use of mask by healthy people in public places has not shown to protect against respiratory viral infections, but WHO recently recommended to wear masks to prevent community transmission if at all present. Public health and social measures to slow or stop the spread of COVID-19 must be implemented with the full engagement of all members of society. Government should consider introducing legislation to prohibit selling and trading of wild animals at the market places.

At the national and international level, strict travel restrictions, evaluation for clinical symptoms at the entry and exit points, isolation and testing for COVID-19 for 2 weeks even if asymptomatic. Contact tracing, tracking of the people who are in contact with the cases, testing of the people for COVID-19, and treatment and hospitalization of the confirmed cases. Guidelines and stringent rules should be made to follow; medical staff, healthcare providers, public health individuals and researchers who are interested in the 2019-nCoV, as per the recommended guidelines by the national, international and state bodies [74].

\section{Conclusions}

This zoonotic pandemic disease caused by nCoV-19 originated from the Hunan seafood trade market at Wuhan, China where wild animals like snakes like cobras, bats, raccoons, dogs, palm civets, and other animals are sold, and it has rapidly spread over to 200 countries. This epidemic outburst has challenged the phys- ical, social, emotional, psychological, economic, medical and public health infrastructure of the whole world even in countries with best of the best healthcare system. Till date, no promising treatments, drugs, mAbs or vaccines or any other prevention strategies, but scientists and researchers around the globe are working hard to develop proficient therapeutic strategies to cope with the $\mathrm{nCoV}$. Various broad-spectrum antivirals, antibiotics and anti-malarial drugs like Remdesivir, Lopinavir, Ritonavir, Oseltamivir, Azithromycin, Chloroquine and HydroxyChloroquine are under trail and significantly blocked the SARSCoV2 infection in COVID-19 patients. Important steps that could curb against the current as well as future epidemics are to be taken to develop, an accurate and rapid diagnostic kit for the detection of SARS-CoV-2 in suspected patients, human CoV targeting vaccines, antiviral drugs, $\mathrm{mAbs}$ and preventive measures. There must be a complete ban on utilizing wild animals and birds as a source of food. Hence, apart from curbing the present epidemic outburst, significant steps should be taken to devise comprehensive broad spectrum actions to prevent future outbreaks of zoonotic origin.

\section{References}

1. Woo PC, Huang Y, Lau SK, Yuen KY. Coronavirus genomics and bioinformatics analysis. Viruses 2010;2:1804-20.

2. Li Q, Guan X, Wu P, et al. Early transmission dynamics in Wuhan, China, of novel coronavirus-infected pneumonia. N Engl J Med 2020;382:1199-207.

3. Huang C, Wang Y, Li X, et al. Clinical features of patients infected with 2019 novel coronavirus in Wuhan, China. Lancet 2020;395 497-506.

4. Chen N, Zhou M, Dong X, et al. Epidemiological and clinical characteristics of 99 cases of 2019 novel coronavirus pneumonia in Wuhan, China: a descriptive study. Lancet 2020;395: 507-13.

5. Wang D, Hu B, Hu C, et al. Clinical characteristics of 138 hospitalized patients with 2019 novel coronavirus-infected pneumonia in Wuhan, China. JAMA 2020;323:1061-9.

6. Liu K, Fang YY, Deng Y, et al. Clinical characteristics of novel coronavirus cases in tertiary hospitals in Hubei Province. Chin Med J (Engl) 2020;133:1025-31.

7. WHO. Coronavirus disease 2019 (COVID-19) Situation Report - 40. Accessed on: 2 April 2020. Available from: https://www. who.int/docs/default-source/coronaviruse/situation-reports/ 20200229-sitrep-40-covid-19.pdf?sfvrsn=849d0665_2

8. Richman DD, Whitley RJ, Hayden FG. Clinical virology.Washington: ASM Press; 2016.

9. Yin Y, Wunderink RG. MERS, SARS and other coronaviruses as causes of pneumonia. Respirology 2018;23:130-7.

10. Wang N, Shi X, Jiang L, et al. Structure of MERS-CoV spike receptor-binding domain complexed with human receptor DPP4. Cell Res 2013 23:986.

11. [No authors listed]. Seven days in medicine: 8-14 Jan 2020. BMJ 2020;368:m132.

12. WHO. Coronavirus disease (COVID-19) technical guidance: Laboratory testing for 2019-nCoV in humans. Available from: https://www.who.int/emergencies/diseases/novel-coronavirus2019/technical-guidance/laboratory-guidance

13. Zhou F, Yu T, Du R, et al. Clinical course and risk factors for mortality of adult in patients with COVID-19 in Wuhan, China: a retrospective cohort study. Lancet 2020;395:1054-62. 
14. WHO. Coronavirus disease (COVID-2019) situation reports. Accessed on: 2 April 2020. Available from: https://www. who.int/emergencies/diseases/novel-coronavirus-2019/situation-reports/

15. Chen H, Guo J, Wang C, et al. Clinical characteristics and intrauterine vertical transmission potential of COVID-19 infection in nine pregnant women: a retrospective review ofmedical records. Lancet 2020;395:809-15..

16. Cheng ZJ, Shan J. 2019 novel coronavirus: where we are and what we know. Infection 2020:48:155-63.

17. Peiris J, Guan Y, Yuen K. Severe acute respiratory syndrome. Nat Med 2004;10:S88-97.

18. Pyrc K, Berkhout B, Van Der Hoek L. Identification of new human coronaviruses. Expert Rev Anti Infect Ther 2007;5: 245-53.

19. Rahman A, Sarkar A. Risk factors for fatal Middle East respiratory syndrome coronavirus infections in Saudi Arabia: Analysis of the WHO Line List, 2013-2018. Am J Public Health 2019;109:1288-93.

20. Memish ZA, Zumla AI, Al-Hakeem RF, et al. Family cluster of Middle East respiratory syndrome coronavirus infections. N Engl J Med 2013;368:2487-94.

21. Wang C, Horby PW, Hayden FG, Gao GF. A novel coronavirus outbreak of global health concern. Lancet 2020;395:470-3.

22. Xinhua. [Internet]. China's CDC detects a large number of new coronaviruses in the South China seafood market in Wuhan. Accessed on: 20 February 2020. Available from: https://www. xinhuanet.com/2020-01/27/c_1125504355.htm

23. Luk HK, Li X, Fung J, et al. Molecular epidemiology, evolution and phylogeny of SARS coronavirus. Infect Genet Evol 2019; $71: 21-30$

24. Wu JT, Leung K, Leung GM. Now casting and forecasting the potential domestic and international spread of the 2019-nCoV outbreak originating in Wuhan, China: A modelling study. Lancet 2020;395:689-97.

25. Zhou P, Yang X, Wang X, et al. A pneumonia outbreak associated with a new coronavirus of probable bat origin. Nature 2020;579:270-3.

26. Wu F, Zhao S, Yu B, et al. A new coronavirus associated with human respiratory disease in China. Nature 2020;579:265-9. Erratum in: Nature 2020;580:E7. doi: 10.1038/s41586-0202202-3

27. Wan Y, Shang J, Graham R, et al. Receptor recognition by novel coronavirus from Wuhan: An analysis based on decadelong structural studies of SARS. J Virol 2020. doi: 10.1128/JVI.00127-20

28. Annan A, Baldwin HJ, Corman VM, et al. Human betacoronavirus 2c EMC/2012-related viruses in bats, Ghana and Europe. Emerg Infect Dis 2013;19:456.

29. Lu R, Zhao X, Li J, et al. Genomic characterisation and epidemiology of 2019 novel coronavirus: implications for virus origins and receptor binding. Lancet 2020;395:565-74.

30. Liu L, Wu P, Gao F, et al. Novel immunodominant peptide presentation strategy: a featured HLA-A*2402-restricted cytotoxic T-lymphocyte epitope stabilized by intrachain hydrogen bonds from severe acute respiratory syndrome coronavirus nucleocapsid protein. J Virol 2010;84:11849-57.

31. Glowacka I, Bertram S, Müller MA, et al. Evidence that TMPRSS2 activates the severe acute respiratory syndrome coronavirus spike protein for membrane fusion and reduces viral control by the humoral immune response. J Virol 2011;85:4122-34.

32. Li G, Chen X, Xu A. Profile of specific antibodies to the
SARS-associated coronavirus. N Engl J Med 2003;349:508-9.

33. Xu Z, Shi L, Wang Y, et al. Pathological findings of COVID19 associated with acute respiratory distress syndrome. Lancet Resp Med 2020;8:420-2.

34. Channappanavar R, Perlman S. Pathogenic human coronavirus infections: causes and consequences of cytokine storm and immunopathology. Semin Immunopathol 2017;39:529-39.

35. Min CK, Cheon S, Ha NY, et al. Comparative and kinetic analysis of viral shedding and immunological responses in MERS patients representing a broad spectrum of disease severity. Sci Rep 2016;6:25359.

36. Snijder EJ, van der Meer Y, Zevenhoven-Dobbe J, et al. Ultrastructure and origin of membrane vesicles associated with the severe acute respiratory syndrome coronavirus replication complex. J Virol 2006;80:5927-40.

37. Menachery VD, Schafer A, Burnum-Johnson KE, et al. MERS-CoV and $\mathrm{H} 5 \mathrm{~N} 1$ influenza virus antagonize antigen presentation by altering the epigenetic landscape. P. Natl. Acad. Sci. USA 2018;115:E1012-21.

38. Wang W, Tang J, Wei F. Updated understanding of the outbreak of 2019 novel coronavirus (2019-nCoV) in Wuhan, China. J Med Virol 2020;92:441-7.

39. Lei J, Li J, Li X, Qi X. CT imaging of the 2019 novel coronavirus (2019-nCoV) pneumonia. Radiology 2020;295:18.

40. Phan LT, Nguyen TV, Luong QC, et al. Importation and human-to-human transmission of a novel coronavirus in Vietnam. N Engl J Med 2020;382:872-4.

41. Lee N, Hui D, Wu A, et al. A major outbreak of severe acute respiratory syndrome in Hong Kong. $\mathrm{N}$ Engl J Med 2003;348:1986-94.

42. Corman VM, Landt O, Kaiser M, et al. Detection of 2019 novel coronavirus (2019-nCoV) by real-time RT-PCR. Euro Surveill 2020;252000045.

43. Zou L, Ruan F, Huang M, et al. SARSCoV-2 viral load in upper respiratory specimens of infected patients. N Engl J Med 2020;382:1177-9.

44. Yam WC, Chan KH, Poon LL, et al. Evaluation of reverse transcription-PCR assays for rapid diagnosis of severe acute respiratory syndrome associated with a novel coronavirus. J Clin Microbiol 2003;41:4521-4.

45. Woo PC, Lau SK, Wong BH, et al. Differential sensitivities of severe acute respiratory syndrome (SARS) coronavirus spike polypeptide enzyme-linked immunosorbent assay (ELISA) and SARS coronavirus nucleocapsid protein ELISA for serodiagnosis of SARS coronavirus pneumonia. J Clin Microbiol 2005;43:3054-8.

46. Huang P, Liu T, Huang L, et al. Use of chest CT in combination with negative RT-PCR assay for the 2019 novel coronavirus but high clinical suspicion. Radiology 2020;295:22-3.

47. Wu Z, McGoogan JM. Characteristics of and important lessons from the coronavirus disease 2019 (COVID-19) outbreak in China: summary of a report of 72314 cases from the Chinese Center for Disease Control and Prevention. JAMA 2020. doi: 10.1001/ jama.2020.2648.

48. Russell CD, Millar JE, Baillie JK. Clinical evidence does not support corticosteroid treatment for 2019-nCoV lung injury. Lancet 2020;395:473-5.

49. Zhao JP, Hu Y, Du RH, et al. Expert consensus on the use of corticosteroid in patients with 2019-nCoV pneumonia. Zhonghuaie He He Hu Xi Za Zhi. 2020;43:E007.

50. WHO. Clinical management of severe acute respiratory infection when novel coronavirus $[\mathrm{nCoV}]$ infection is suspected. Accessed on: 2 April 2020. Available from: https://www. 
who.int/publications-detail/clinical-management-of-severeacute-respiratory-infection-when-novel-coronavirus-(ncov)infection-is-suspected

51. National Institute of Allergy and Infectious Diseases (NIAID) (US). Identifier NCT04280705, Adaptive COVID-19 Treatment Trial (ACTT). 2020 Feb 21. Available from: https://clinicaltrials.gov/ct2/show/NCT04280705.

52. Cao B, Wang Y, Wen D, et al. A trial of lopinavir-ritonavir in adults hospitalized with severe Covid-19. N Engl J Med 2020;382):1787-99.

53. Wang BX, Fish EN. Global virus outbreaks: Interferons as 1st responders. Semin Immunol 2019; 43:101300.

54. Wang M, Cao R, Zhang L, et al. Remdesivir and chloroquine effectively inhibit the recently emerged novel coronavirus (2019-nCoV) in vitro. Cell Res 2020:30:269-71.

55. Agostini ML, Andres EL, Sims AC, et al. Coronavirus susceptibility to the antiviral remdesivir (GS-5734) is mediated by the viral polymerase and the proofreading exoribonuclease. MBio 2018; 9:e00221-18.

56. Sheahan TP, Sims AC, Leist SR, et al. Comparative therapeutic efficacy of remdesivir and combination lopinavir, ritonavir, and interferon beta against MERS-CoV. Nat Commun 2020;11:22.

57. Holshue ML, DeBolt C, Lindquist S, et al. First case of 2019 novel coronavirus in the United States. $N$ Engl J Med 2020;382:929-36.

58. Richardson P, Griffin I, Tucker C, et al. Baricitinib as potential treatment for 2019-nCoV acute respiratory disease. Lancet 2020;395:e30-1.

59. Ye Z, Zhang Y, Wang Y, et al. Chest CT manifestations of new coronavirus disease 2019 (COVID-19): a pictorial review. Eur Radiol 2020. doi.: 10.1007/s00330-020-06801-0.

60. Teachey DT, Lacey SF, Shaw PA, et al. Identification of predictive biomarkers for cytokine release syndrome after chimeric antigen receptor T-cell therapy for acute lymphoblastic leukemia. Cancer Discov 2016;6:664-9.

61. Lu H. Drug treatmentoptions for the 2019-newcoronavirus (2019-nCoV). Biosci Trends 2020;14:69-71.

62. Zhang JS, Chen JT, Liu YX, et al. A serological survey on neutralizing antibody titer of SARS convalescent sera. J Med Virol 2005; 77:147-5.

63. Beigel JH, Voell J, Kumar P, et al Safety and tolerability of a novel, polyclonal human anti-MERS coronavirus antibody produced from transchromosomic cattle: a phase 1 ran- domised, double-blind, single-dose-escalation study. Lancet Infect Dis 2018;18 410-8.

64. Shen C, Wang Z, Zhao F, et al. Treatment of 5 critically ill patients with COVID-19 with convalescent plasma. JAMA 2020;323:1582-9.

65. Derebail VK, Falk RJ. ANCA-associated vasculitis-refining therapy with plasma exchange and glucocorticoids. N Engl J Med 2020;382:671-3.

66. Tian X, Li C, Huang A, et al. Potent binding of 2019 novel coronavirusspike protein by a SARS coronavirus-specific human monoclonal antibody. Emerg Microbes Infect 2020;9:382-5.

67. Bisht H, Roberts A, Vogel L, et al. Neutralizing antibody and protective immunity to SARS coronavirus infection of mice induced by a soluble recombinant polypeptide containing an $\mathrm{N}$-terminal segment of the spike glycoprotein. Virology 2005;334:160-5.

68. Kam YW, Kien F, Roberts A, et al. Antibodies against trimeric $\mathrm{S}$ glycoprotein protect hamsters against SARS-CoV challenge despite their capacity to mediate Fc $\gamma$ RII-dependent entry into B cells in vitro. Vaccine 2007;25:729-40.

69. Wall Street Journal [Internet]. Drugmakers rush to develop vaccines against china virus. Accessed on: 28 January 2020. Available from: https:/www.wsj.com/articles/drugmakersrushto- develop-vaccines-against-china-virus-11579813026

70. PR Newswire [Internet]. Inovio selected by cepi to develop vaccine against new coronavirus inovio. Available from: http://ir.inovio.com/news-and-media/news/pressreleasedetails/2020/Inovio-Selectedby-CEPI-to-Develop-VaccineAgainstNewCoronavirus/default.aspx

71. Clover Biopharmaceuticals. Clover initiates development of recombinant subunit- trimer vaccine for Wuhan coronavirus (2019-nCov). Accessed on: 6 march 2020. Available from: http://www.cloverbiopharma.com/index.php? $\mathrm{m}=$ content\&c=in dex\&a $=$ show $\&$ catid $=11 \& i d=40$

72. Chang D, Xu H, Rebaza A, et al. Protecting health-care workers from subclinical coronavirus infection. Lancet Respir Med 2020;8:e13.

73. Centers for Disease Control and Prevention (CDC). 2019 Novel Coronavirus. How to protect yourself \& others. Available from: https://www.cdc.gov/coronavirus/2019ncov/prevent-getting-sick/prevention.html

74. Jin YH, Cai L, Cheng XS, et al. A rapid advice guideline for the diagnosis and treatment of 2019 novel coronavirus (2019$\mathrm{nCoV}$ ) infected pneumonia. Milit Med Res 2020;7:4. 